\title{
Change perspective to increase diagnostic accuracy of ultrasonography in calcium pyrophosphate dihydrate deposition disease! A new approach: the axial scan of the meniscus
}

\author{
G. Filippou, V. Picerno, A. Adinolfi, V. Di Sabatino, I. Bertoldi, M. Galeazzi, \\ B. Frediani \\ Department of Medicine, Surgery and Neurosciences, Rheumatology Unit, University of Siena, Italy
}

\section{SUMMARY}

Ultrasonography (US) is a relevant tool in the study of calcium pyrophosphate dihydrate (CPP) deposition disease. However, differential diagnosis of hyperechoic deposits within the fibrocartilage can be difficult; moreover, US study is limited by the need of an adequate acoustic window.

We describe a US scanning technique that offers a new viewpoint in the study of knee meniscal structure: a longitudinal scan performed according to the long axis of meniscus. This technique proves to be particularly useful for the identification of CPP deposition, but could also improve the US diagnostic utility and accuracy in other meniscal pathologies.

Key words: CPPD; chondrocalcinosis; knee; meniscus; ultrasound.

Reumatismo, 2014; 66 (4): 318-321

\section{Traditional ultrasonographic study of the meniscus in calcium pyrophosphate dihydrate deposition disease: why and how}

Ultrasound is a useful tool for the study of joint and soft tissue pathology and in the last decade it has been increasingly used by Rheumatologists as a routine exam in the daily clinical practice. Indeed, thanks to the continuous technologic and software evolution, the last generation US equipment is able to reach real high spatial resolution allowing a precise identification of initial alterations in the joints of Rheumatologic patients. In the last years, the attention of Rheumatologists - sonographers has been focused on the study of fibrocartilage and hyaline cartilage and it has been demonstrated that these two tissues are important either for prognostic purposes $(1,2)$ or, more interestingly, in order to perform diagnosis, especially regarding the microcrystalline arthritides (3-5).
It has been demonstrated actually that calcium pyrophosphate dihydrate (CPP) crystals are most frequently found in the meniscus of patients affected by CPPD (3-5) and it has been suggested that this structure could be the sentinel of the disease (4). However it has also been demonstrated that meniscus is not easy to scan and there are some pitfalls that have to be known and avoided (6).

Sonographic scans generally used to evaluate knee menisci are medial and lateral scans in the longitudinal axis of the femoral-tibial joint performed with the knee in neutral position and in slight flexion $\left(30^{\circ}\right)$ $(7,8)$. Normal lateral and medial meniscus appear as triangular echoic structures between femoral and tibial margins, with the basis immediately under the collateral ligaments and the apixes pointing toward the center of the joint. Valgus and varus stress of the joint can increase the visibility of anterior horn of medial and lateral 
menisci (8), while dorsal scans, with the patient in prone position, can be performed for the evaluation of the posterior horn (8). The main features of CPP deposition in the meniscus, as seen in the classic transverse view has been previously described and the sensitivity and specificity of these findings for the diagnosis of CPPD have been assessed (3-5).

But also other sonographic alterations of the meniscal structure can be observed in osteoarthritis of the knee, related to fibrocartilage degeneration and dehydration (9, 10 ); fibrosis can be visualized as hyperechoic areas, elongated or oval shaped, depending on their position and orientation in the meniscal structure. In late phases of the disease, severe meniscal thinning and lateral dislocation until complete extrusion can be observed.

Meniscal tears appear at the sonographic evaluation as linear defects of the meniscal structure that generally reach at least one of the free margins of the fibrocartilage; they can appear as hyperechoic lines or as hypoechoic or anechoic clefts, if meniscal fragments are displaced and synovial fluid enters in the structure (10). Cystic formations can be visualized in connection with menisci, related to meniscus lesion or degeneration (10).

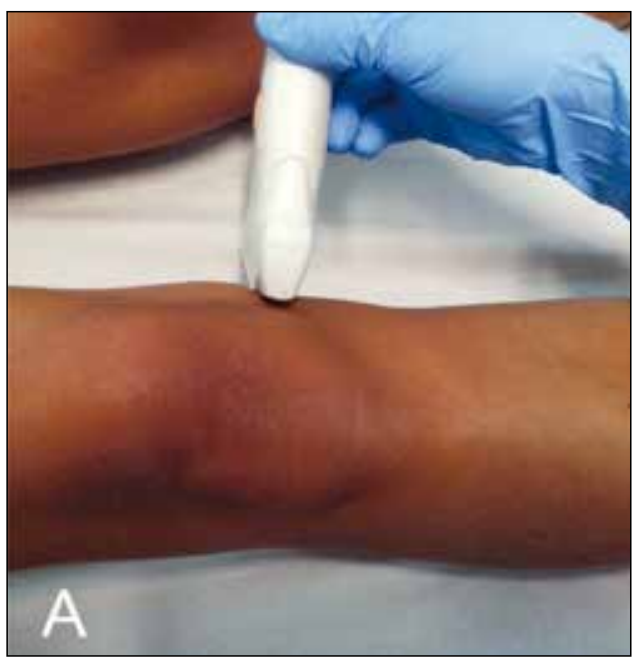

\section{Longitudinal scanning of meniscus in calcium pyrophosphate dihydrate deposition disease: how and when}

In a previous study (6) on the sonographic diagnosis of CPPD in patients who underwent knee replacement surgery for osteoarthritis, we observed some inconsistencies between the in vivo and ex vivo sonographic exams of knee menisci, conducted before and after joint surgery. This observation allowed us to highlight some difficulties in CPP crystals detection by ultrasonography (US) and also to propose some technical tips in order to improve the sonographic approach to CPPD identification in fibrocartilage.

However, CPP identification remains challenging even for expert sonographers. For example, areas of fibrosis may appear with the same sonographic aspect of CCP deposits (small round shaped and hyperecoic areas) on the basis of their position and orientation in the meniscus making the operator not able to distinguish the two conditions. False positive images of CPP deposits can sometimes be due to the posterior enhancement created by small vessels in the meniscus or by the anechoic cleft of a meniscal lesion. Finally, in case of severe osteoarthritis, advanced meniscus degeneration with loss of the typical

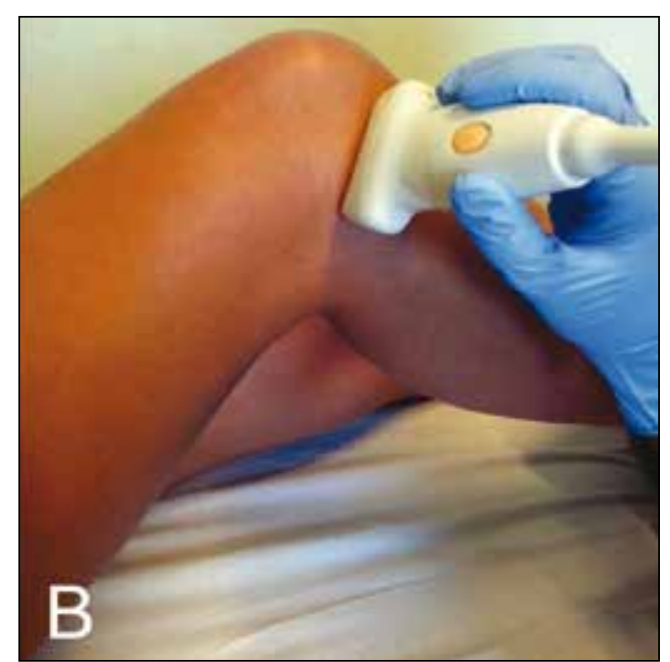

Figure 1 - Longitudinal scan of a meniscus: probe is oriented according the long axis of the fibrocartilage structure just between epiphyseal extremities of femur and tibia with knee extended $(A)$ or in slight flexion (B). Both scans could be performed either for medial $(A)$ or lateral (B) meniscus. 

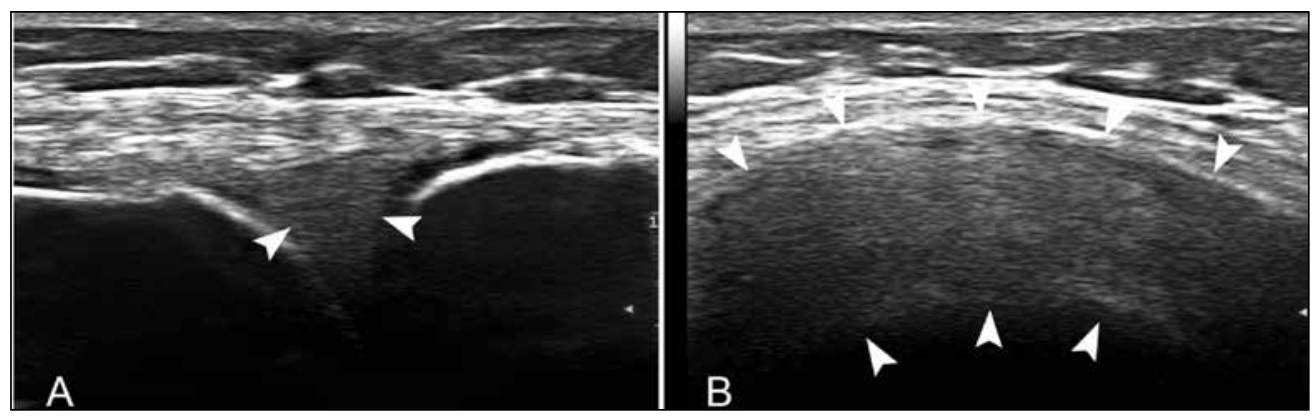

Figure 2 - Transversal (A) and longitudinal (B) scans of medial meniscus in a healthy knee: longitudinal scan allows a more complete view of the fibrocartilage structure that appears as a hyperechoic elongated body between superficial and deep margins (tips of arrows).

triangular shape and marked inhomogeneity make detection of deposits more difficult; only a part of meniscal fibrocartilage could be adequately visualized, with the risk of missing deposits that could facilitate diagnosis.

In some of these cases, a new viewpoint could be a real eye-opener for the diagnosis. The longitudinal scanning of the meni- scus (according to the meniscus long axis and not to the joint long axis) can offer this new perspective.

This scan can be performed with the knee extended or in slight flexion, positioning the probe on the lateral and medial side of the joint just between the femoral and tibial extremities with the long axis of the probe parallel to the long axis of the me-

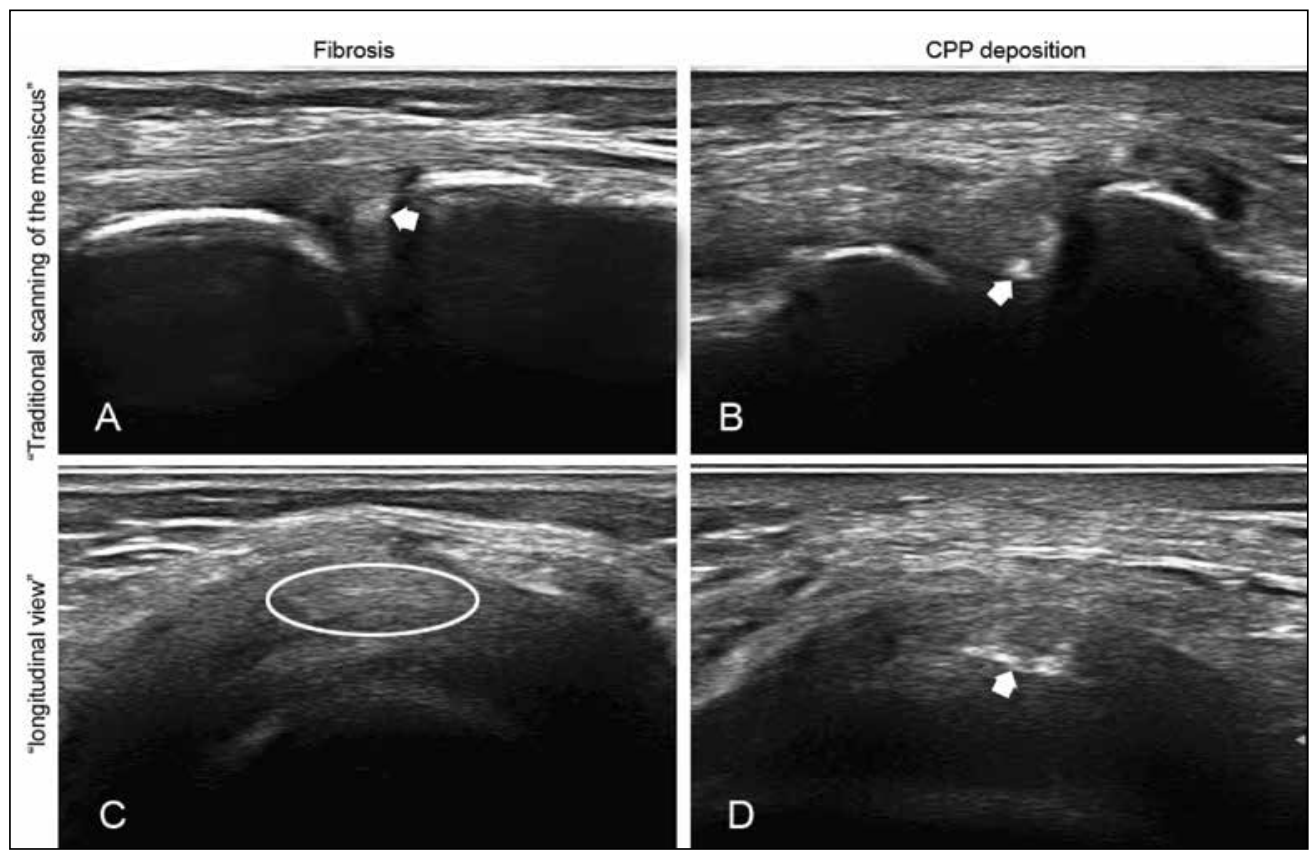

Figure 3 - Detection of fibrosis and calcium pyrophosphate dihydrate deposition disease (CPPD) deposits in medial menisci. In (A) transversal scan of medial meniscus, the presence of round echoic area due to fibrosis, resembling crystal deposits can perturb the observer; longitudinal view $(C)$ clarifies the nature of formation as elongated, inhomogeneous area of fibrosis. In B and D detection of CPPD deposits on transversal (B) and longitudinal (D) scans of meniscus: the longitudinal view allows to detect confluent deposits form a continuous line that would be missing in the traditional scan. 
niscus (Figure 1). Meniscus fibrocartilage can be visualized as an echoic, elongated structure and its whole surface can be analyzed with a continuous scan, sliding from the anterior to the posterior horn without raising the probe (Figure 2).

In our observation, longitudinal scan of the meniscus can be useful for clarifying the nature of meniscal hyperechoic deposition, allowing to better identify CPPD. CPP crystal deposition in longitudinal scanning appears generally as linear or oval/round shaped deposits that tend to be confluent and in some cases to form a continuous line parallel to the meniscus surface.

This aspect is very similar to what we observe in the hyaline cartilage.

On the other hand, fibrosis appears as long and generally continuous parallel line, slightly hyperechoic in comparison with the normal meniscus but hypoechoic in comparison with CPP deposition (Figure 3 ). In some cases the presence of synovial fluid within meniscal tears can mimic the presence of CPPD in the meniscus as it can create hyperechoic interfaces.

With the longitudinal scanning, application of pressure on the meniscus is possible (there is no bone as in the traditional scan) and the movement of the synovial fluid can facilitate the differential diagnosis.

\section{CONCLUSIONS}

Scanning of the meniscus is very important for the diagnosis of CPPD as this is the most frequently involved structure in this disease.

The scanning method proposed in this paper could aid to further increase the diagnostic accuracy of US in CPPD. Further, this new scanning technique could be also used and studied in other meniscal pathologies (i.e. tears) as this new window on the meniscus could improve the US diagnostic utility and accuracy in various pathologic conditions of this structure.

\section{REFERENCES}

1. Filippucci E, da Luz KR, Di Geso L, Salaffi F, Tardella M, Carotti M, et al. Interobserver reliability of ultrasonography in the assessment of cartilage damage in rheumatoid arthritis. Ann Rheum Dis. 2010; 69: 1845-8.

2. Iagnocco AM, Conaghan PG, Aeqerter P, Möller I, Bruyn GA, Chary-Valckenaere I, et al. The reliability of musculoskeletal ultrasound in the detection of cartilage abnormalities at the metacarpo-phalangeal joints. Osteoarthritis Cartilage. 2012; 20: 1142-6.

3. Filippou G, Frediani B, Gallo A, Menza L, Falsetti P, Baldi F, et al. A "new" technique for the diagnosis of chondrocalcinosis of the knee: sensitivity and specificity of highfrequency ultrasonography. Ann Rheum Dis. 2007; 66: 1126-8.

4. Filippou G, Filippucci E, Tardella M, Bertoldi I, Di Carlo M, Adinolfi A, et al. Extent and distribution of CPP deposits in patients affected by calcium pyrophosphate dihydrate deposition disease: an ultrasonographic study. Ann Rheum Dis. 2013; 72: 1836-9.

5. Filippucci E, Di Geso L, Girolimetti R, Grassi W. Ultrasound in crystal-related arthritis. Clin Exp Rheumatol. 2014; 32; S42-7.

6. Filippou G, Adinolfi A, Bozios P, Lorenzini S, Picerno V, Di Sabatino V, et al. Do not hallow until you are out of the wood! Ultrasonographic detection of CPP crystal deposits in menisci: facts and pitfalls. Sci World J. 2013; 16: 181826.

7. Backhaus M, Burmester GR, Gerber T, Grassi W, Machold KP, Swen WA, et al. Guidelines for musculoskeletal ultrasound in rheumatology. Ann Rheum Dis. 2001; 60: 641-9.

8. Bianchi S, Martinoli C. Ultrasound of the musculoskeletal system. Berlin: Springer Verlag; 2007.

9. Iagnocco AM, Perricone C, Scirocco C. The interobserver reliability of ultrasound in knee osteoarthritis. Rheumatology. 2012; 19: 2013-9.

10. Court-Payern M. Sonography of the knee: intra-articular pathology. J Clin Ultrasound. 2004; 32: 481-90. 